


\title{
Sentimentos vivenciados por fisioterapeutas no atendimento a pessoas com paralisia cerebral
}

\author{
Dinael Corrêa de Campos ${ }^{1}$ - Universidade São Francisco, São Paulo, Brasil \\ Márcia Guimarães Santos ${ }^{2} 3$ - Universidade São Francisco, São Paulo, Brasil
}

\begin{abstract}
Resumo
Considerando que os estudos sobre a relação profissional de saúde e cliente contribuíram para o surgimento da proposta do modelo humanizado de atendimento, esta pesquisa investigou os possíveis sentimentos de fisioterapeutas em relação ao atendimento de pessoas com paralisia cerebral, já que esta condição, a paralisia cerebral, é considerada sem cura pela medicina e com possibilidades de melhora diretamente vinculadas aos exercícios fisioterapêuticos. Utilizando um instrumento contendo perguntas abertas, foram realizadas entrevistas com fisioterapeutas que atendem aos casos com sequelas mais graves: ausência total da linguagem verbal e comprometimentos motores que levam os pacientes à situação de acamados. Mediante a análise de conteúdo, verificou-se que a paralisia cerebral é reconhecida pelos fisioterapeutas como um quadro neurológico e as limitações no alcance de resultados terapêuticos são vistas como normais. Assim, as mudanças, mesmo sendo reduzidas e obtidas em longo prazo, tornam o atendimento gratificante para os fisioterapeutas por representarem uma superação de desafios.

Palavras-chave: saúde humanizada; fisioterapia; paralisia cerebral.
\end{abstract}

\section{Physiotherapist's feelings on attending people with cerebral palsy}

\begin{abstract}
Considering the studies about professional relation among health and clients contributted to arise the purposes about a humanized model of assistance. This research shows the physiotherapists' feelings on attending people with cerebral palsy, taking in accounting the cerebral palsy is presented by the medicine as a reverseless decease and having possibilities of changing entailed to the physiotherapy. By means of interviews and questionaries answered by physiotherapists, and using the contents of analysis, we can notice that the cerebral palsy is understood by the professional of physiotherapy like a neurological condition, so the limitations on reaching are noticed as normal. Thus, even the changes reduced in the long run, they make the attendance to be rewarding for the professional of physiotherapy, because these changes represent the overcoming of the challenge.

Keywords: humanization health; physiotherapist; cerebral palsy.
\end{abstract}

\section{Introdução}

$\mathrm{Na}$ atualidade, intensificaram-se os estudos sobre as vivências dos profissionais no contexto da saúde. Diversos autores como Nogueira-Martins (2004) e Takka (1988) têm apontado as angústias vividas por profissionais e estudantes de saúde em relação ao atender, sendo as mesmas relacionadas ao fato de lidarem com a dor, a morte e a invalidez objetos de trabalho desses profissionais:

As angústias ligadas ao adoecer são parte integrante dessa relação, que é o campo dinâmico que se estabelece entre o cliente e aquele que o assiste, onde surgem as angústias básicas profundas e intensas que se desenvolvem no vínculo estabelecido. (Nogueira-Martins, 2004, p. 8)

Assim, a crescente preocupação em investigar tais vivências do ponto de vista dos profissionais e estudantes, tem revelado os aspectos humanos do profissional que devem ser considerados para uma humanização da assistência, uma vez que esses aspectos se revelam como caracterizadores do tipo de relações que se estabelecem entre um profissional de saúde e seus clientes. Além disso, Takka (1988) ressalta que a recuperação de um paciente pode ser notadamente mais rápida quando há um relacionamento apropriado entre este e o médico.

A humanização dos serviços de saúde é a proposta de um modelo de atendimento que procura aliar os conhecimentos técnicos a uma prática assistencial que considere os direitos e a subjetividade dos clientes (Deslandes, 2004). Sendo assim, humanizar os serviços ressalta a importância de considerar a dimensão relacional existente entre os profissionais de saúde e seus clientes, exigindo mudanças, tanto em relação aos modelos de atendimentos que têm privilegiado o tecnicismo em detrimento da qualidade das relações humanas no contexto da saúde quanto no que se refere às

\footnotetext{
${ }^{1}$ Docente orientador da pesquisa

${ }^{2}$ Discente autora da pesquisa

${ }^{3}$ Endereço para correspondência:

R. Ibiaí, 26, Parque Alvorada - 07242-280 - Guarulhos-SP, Brasil

E-mail: gmarciam@yahoo.com.br
} 
atitudes dos profissionais em relação aos usuários dos serviços (Deslandes \& Ayres, 2005).

Os resultados dos estudos de Nogueira-Martins (2004), Rocco (1992) e Takka (1988), aliados à proposta de humanização da assistência, têm instigado críticas aos currículos aplicados nos cursos de formação de profissionais em saúde, uma vez que os mesmos continuaram difundindo o modelo biomédico de atendimento, caracterizado por enxergar o ser humano fragmentado, que para ser atendido requer que seja dividido em partes, resultando de tal prática a crescente divisão das profissões de saúde em especializações. Especificamente aqui nos interessa o curso de fisioterapia, e Ricieri (2003, apud Braz, 2006) afirma que:

[...] tanto o ensino quanto a prática da Fisioterapia ainda seguem os modelos adotados pela Medicina: a fragmentação do ser humano, a divisão em especialidades, a ênfase na cura e na reabilitação (a recuperação ou "conserto" da parte afetada) e o tecnicismo, amparados pelo modelo da física clássica.

Segundo Braz (2006), os críticos desse paradigma afirmam que é por carregar esta visão sobre o ser humano que tal modelo não atende às expectativas da proposta de assistência humanizada, pois esta pressupõe a consideração do ser humano de uma forma mais integrada, que abarque suas dimensões biológica, psicológica, social, ética, cultural e espiritual. E é com base nesse contexto, apresentado por Braz (2006), que o presente estudo qualitativo elege, para fins investigativos, os profissionais de fisioterapia, uma vez que tal profissional, em sua prática, tem a oportunidade de prestar um atendimento integrado junto às pessoas com paralisia cerebral.

A fisioterapia é definida pelo COFFITO Conselho Federal de Fisioterapia e Terapia Ocupacional, órgão regulamentador da profissão, como uma ciência da saúde que se ocupa do movimento humano, em estudá-lo, tratá-lo e prevenir os distúrbios a ele relacionados, sejam estes originados pela genética, por traumatismos ou patologias adquiridas.

O fisioterapeuta é o profissional da área da saúde que tem formação no ensino superior, cuja atividade permite que realize diagnóstico dos distúrbios do movimento humano, a prescrição dos procedimentos fisioterapêuticos indicados e o acompanhamento do quadro, e tem seu exercício profissional fiscalizado pelo Conselho Regional de Fisioterapia e Terapia Ocupacional, o CREFITO, órgão regional designado à legalização do exercício profissional e fiscalização do cumprimento das leis que regulam as atuações profissionais de fisioterapeutas e terapeutas - ocupacionais.

Dentre as diversas áreas e os inúmeros tipos de distúrbios do movimento humano nos quais os fisioterapeutas podem atuar, este estudo aborda profissionais de fisioterapia que trabalham com pessoas com paralisia cerebral. A respeito das pessoas com paralisia cerebral que recebem atendimento em fisioterapia é conveniente ressaltar que quanto mais cedo houver a procura ao atendimento fisioterápico, menos comprometimentos físicos poderão ser encontrados.

Basil (1995) define a paralisia cerebral como um termo que abrange diversos distúrbios ocasionados por lesões encefálicas que possam ter ocorrido durante os períodos pré, peri ou pós-natal (ainda na primeira infância). Conforme a autora, a lesão afeta o desenvolvimento motor, podendo causar a alteração ou perda do controle da motricidade, distúrbios no desenvolvimento da fala, distúrbios sensoriais e perceptivos e até mesmo prejuízos na área da inteligência, podendo ocasionar deficiência intelectual grave. Além disso, segundo Diniz (1996), a paralisia cerebral é considerada um quadro irreversível pela medicina atual, sendo seu tratamento mais restrito aos exercícios fisioterápicos do que às intervenções médicas propriamente ditas. Quanto às relações que se estabelecem entre uma pessoa nessa condição e as outras de seu convívio, Basil (1992, p. 260) destaca que:

[...] a falta de percepção de eficácia pode afetar tanto a criança como as pessoas que a cercam, as quais podem ter aprendido que seus esforços são inúteis para conseguirem uma interação apropriada com a criança com problemas motores.

Basil ainda afirma que o tratamento do referido quadro suscita sentimentos de frustração nos fisioterapeutas, considerando também que, em sua formação, normalmente se privilegia o modelo médico voltado para a cura dos sintomas. Outra possível dificuldade experimentada por esses profissionais pode se referir às dificuldades de expressão desses pacientes, caracterizadas por ausência da fala e/ ou limitações motoras graves, fatores que exigem do terapeuta uma flexibilidade para lidar com questões comunicacionais para as quais o mesmo pode não ter sido treinado durante sua formação. Além disso, Diniz (1996, p. 349) descreve os aspectos observáveis numa pessoa com paralisia cerebral grave e as sensações de quem a observa: 
As consultas médicas, nos ambulatórios de pacientes portadores de paralisia cerebral grave, são momentos durante os quais a tristeza e o silêncio reinam. $O$ paciente não fala, não anda, sofre de um retardo mental severo, em resumo, não estabelece quase nenhum vínculo com a realidade. Várias vezes tive a sensação de serem crianças que pareciam olhar através de nós. O quadro da enfermidade sugere, até mesmo ao mais leigo dos observadores, a questão, talvez das mais profundas feitas pela espécie humana, do que venha a ser vida.

Este trabalho se determina a uma dimensão psicológica a ser investigada a respeito da experiência vivida por fisioterapeutas em atender pessoas com paralisia cerebral. A dimensão averiguada é a dos sentimentos, baseada na definição empregada por Dalgalarrondo (2000, p. 101), onde:

os sentimentos são estados e configurações afetivas estáveis; em relação às emoções, são mais atenuados em sua intensidade e menos reativos a estímulos passageiros.

Tendo em vista a importância atribuída ao trabalho do fisioterapeuta junto às pessoas com paralisia cerebral (e as particularidades deste quadro quanto às características e possibilidades de melhora) $\mathrm{e}$ às decorrentes propostas de humanização dos serviços dos profissionais da saúde, esta pesquisa teve como objetivo geral descrever os possíveis sentimentos experimentados por fisioterapeutas ao atenderem pessoas com paralisia cerebral.

A escolha deste tema se deveu ao fato e a pesquisadora, mediante a pesquisa bibliográfica, ter constatado a ausência de publicações científicas contemplando-o, apesar de sua relevância para a atualidade, bem como a duas questões norteadoras que se fizeram presentes para serem investigadas: a possível frustração dos fisioterapeutas em relação ao sucesso do processo de reabilitação e a dificuldade do fisioterapeuta em se comunicar com a pessoa com paralisia cerebral. Sendo assim, considera-se também que esta investigação possa contribuir com o vasto campo de pesquisas científicas na atualidade que se ocupam de compreender as relações terapêuticas do ponto de vista dos profissionais nela envolvidos.

\section{Método}

\section{Participantes}

Participou deste estudo exploratório um grupo de fisioterapeutas que se encontra desenvolvendo atividades de fisioterapia há pelo menos três (3) anos em uma instituição e que durante todo o período atenderam e atendem pessoas com paralisia cerebral grave, pacientes que passam a maior parte do tempo acamados e apresentam ausência total da linguagem verbal.

O grupo de fisioterapeutas em questão foi composto por cinco (5) participantes, número que corresponde ao total de fisioterapeutas atuantes no local, sendo dois (2) do sexo masculino e três (3) do sexo feminino. O tempo médio de formação dos participantes é de cinco anos (2 fisioterapeutas com 4 anos de formação, 1 com 5 anos, 1 com 6 anos e 1 com 7 anos de formação).

Local

A coleta de dados da pesquisa foi desenvolvida nas dependências da instituição, localizada na cidade de São Paulo, estado de São Paulo. Trata-se de uma unidade de longa permanência, servindo, portanto, de abrigo para as pessoas com deficiência intelectual e física associadas.

\section{Instrumento}

O instrumento de coleta de dados foi composto por questões abertas, para que permitisse ao participante responder com maior liberdade, tornando o conteúdo das respostas mais rico e detalhado.

Os dados coletados foram avaliados de forma qualitativa, pois, conforme citado por NogueiraMartins (2004, p. 15): “A metodologia qualitativa tem se mostrado a mais adequada para abordar questões que envolvem percepções, valores, sentimentos e emoções." Sendo assim, considerou-se que as questões abertas forneceriam uma maior quantidade de dados, possibilitando melhor compreensão do fenômeno estudado. Conforme afirma Campos (2003, p. 38) sobre o uso de tal técnica:

[...] a utilização de tal técnica possibilita ao pesquisador organizar e compreender as respostas através de um processo contínuo de identificar dimensões, categorias, tendências, padrões, relações na possibilidade de se desvendar o significado das respostas.

Com base nesses pressupostos e na revisão bibliográfica foi elaborado um questionário com sete (7) questões abertas, que objetivaram adquirir informações sobre os sentimentos experimentados por esses fisioterapeutas durante os atendimentos prestados pelos mesmos às pessoas com paralisia cerebral. As questões foram: 
1-Há quanto tempo você exerce a profissão de fisioterapeuta?

2-Quais motivos te levaram a escolher atuar junto a pessoas com paralisia cerebral?

3-Para você, como é lidar com uma pessoa com paralisia cerebral?

4-Para você, o que é atender um paciente nesta condição?

5-Surgem alguma(s) impressão(s) no seu contato físico (terapêutico) com as pessoas nesta condição, em relação ao que elas estejam sentindo? Se sim, qual(is)? 6-Como é, para você, atender uma pessoa com paralisia cerebral?

7-Tem algum caso de pessoa com paralisia cerebral na sua família?

Para que o pesquisador pudesse manter o sigilo e preservar o anonimato dos entrevistados, a identificação do entrevistado correspondente a cada questionário se deu da seguinte forma: colocava-se a letra "P" (designando o nome "participante"), seguida de um número identificador do participante (exemplo: o participante 1 está identificado no questionário como "P1"; o participante 2 está identificado como "P2", e assim por diante). Esses números seguiram a ordem na qual as entrevistas foram realizadas.

\section{Procedimento}

A pesquisadora entrou em contato pessoal com cada um dos participantes da pesquisa, e nessas ocasiões marcou as datas, os horários e informou o local (sendo este último uma sala nas dependências do prédio administrativo dentro da própria instituição na qual os participantes desenvolvem suas atividades profissionais), onde se reuniu posteriormente com cada profissional de forma individual para a realização das entrevistas de coleta de dados. As perguntas foram respondidas uma a uma, sendo as respostas anotadas para posterior transcrição das mesmas.

$\mathrm{Na}$ ocasião de cada entrevista individual, foram fornecidos aos profissionais os devidos esclarecimentos sobre o tema da referida pesquisa, e os mesmos foram convidados a participar. Em seguida, foram apresentados e entregues aos profissionais os Termos de Consentimento Livre e Esclarecido, para que eles lessem e esclarecessem quaisquer dúvidas que tivessem sobre o termo e a pesquisa.

Após o esclarecimento de todas as dúvidas dos participantes e as devidas assinaturas dos Termos de Consentimento Livre e Esclarecido, na mesma ocasião foram realizadas as entrevistas de forma individual com cada um dos profissionais participantes. Após o encerramento de cada entrevista, a pesquisadora leu as respostas para os participantes, para que não houvesse dúvidas acerca do que foi respondido por eles e anotado pela pesquisadora.

\section{Análise dos dados}

Os dados foram analisados mediante análise de Conteúdo, definida por Vergara (2005, p. 15) da seguinte forma: "a análise de conteúdo é considerada uma técnica para o tratamento de dados que visa identificar o que está sendo dito a respeito de determinado tema."

As perguntas abertas forneceram pelas respostas dos participantes aos pesquisadores, uma maior quantidade de dados, com maior riqueza de detalhes sobre o que se desejava investigar. Campos (2003) afirma que isso pode dificultar a análise do material coletado, pois pode ser que na resposta a uma pergunta aberta sejam encontradas mais de uma idéia, o que exigirá maior acuidade por parte do pesquisador.

Dessa forma, a análise de conteúdo apresentou-se como um método adequado para esse tipo de investigação, já que propõe, obrigatoriamente, que os dados sejam subdivididos em unidades e categorias para que possam ser analisados (Vergara, 2005).

Por se tratar de uma pesquisa de caráter exploratório, foi utilizada a grade aberta, na qual o pesquisador definiu as categorias de análise durante a execução da pesquisa, conforme recomendado por Vergara (2005). Isso quer dizer que as categorias foram definidas de acordo com as que foram encontradas no material coletado, após a leitura das respostas dadas pelos participantes às questões. Esse tipo de procedimento permitiu agrupar elementos semelhantes nos discursos de diferentes sujeitos. Para realizar tal procedimento, a tabulação contemplou a idéia básica de cada resposta dada às questões, uma vez que:

quando se utiliza a pergunta aberta, [...], a tabulação não é feita considerando a pergunta como um todo, pois, quanto mais rica elas forem, mais difícil será encontrar uma resposta exatamente igual à outra. (Campos, 2003, p. 42).

Com a finalidade de evitar a interferência da subjetividade do pesquisador na definição das categorias, e para garantir a neutralidade que confere a um trabalho de cunho científico, o pesquisador adotou dois (2) juízes para a análise do conteúdo das 
respostas, para evitar o que Minayo (1996, p. 197) denominou de "ilusão da transparência":

[...] 'ilusão da transparência' isto é, o perigo da compreensão espontânea como se o real se mostrasse nitidamente ao observador. Trata-se de uma luta contra a sociologia ingênua e o empirismo, que acreditam poder apreender as significações dos atores sociais, mas apenas conseguem a projeção de sua própria subjetividade.

Sendo assim, o pesquisador procedeu da seguinte forma, sugerida por Campos (2003): os juízes separadamente analisaram as respostas às perguntas do questionário e ressaltaram as "falas" que julgaram mais pertinentes em relação ao tema da pesquisa, ou seja, o pesquisador reproduziu cópias de todas as respostas da forma como foram respondidas e encaminhou todo o material para os outros dois juízes que, separadamente, analisaram as respostas, ou seja: os pesquisadores leram todas as respostas à primeira pergunta e assinalaram o conteúdo que se repetia nas respostas dos participantes, e assim se procedeu para cada pergunta/resposta. Os juízes foram orientados a adotarem o mesmo procedimento, ou seja, assinalar o conteúdo que segundo cada um deles, se repetia. Com tal procedimento, a repetição de respostas formou uma unidade para se caracterizar um tema de estudo.

Posteriormente, os juízes remeteram aos pesquisadores suas respostas assinaladas e o mesmo procedeu da seguinte forma: quando houve $\mathrm{O}$ assinalamento das mesmas respostas por parte de um dos dois juízes, essas respostas foram consideradas significativas e aptas a formarem um tema. Com esse procedimento de análise adotado, acredita-se que os dados foram tratados de forma cuidadosa e específica no que se refere ao conteúdo das falas dos participantes da pesquisa, não incorrendo nos seguintes riscos apontados por Minayo (1996, p. 198):

[...] freqüentemente oculta aquele estado nebuloso, límbico, no trabalho de pesquisa social, que pode ao mesmo tempo esconder alta criatividade e procedimentos analíticos pouco confiáveis técnica e cientificamente.

Após a classificação de todas as falas apontadas pelos juízes, o pesquisador classificou as mesmas em uma ou mais categorias que julgou conveniente e as entregou novamente aos dois juízes, buscando a concordância dos mesmos. O procedimento solicitou a anuência ou não da classificação das falas nas categorias propostas, onde se buscou, com tal procedimento, dirimir a subjetividade do pesquisador.

Os procedimentos adotados e descritos possibilitaram a análise temática de cada uma das respostas emitidas pelos participantes e classificadas pelos juízes da pesquisa, e que foram analisadas tendo em vista a tabulação conforme a classificação das respostas.

\section{Discussão dos resultados}

A análise dos dados coletados permitiu eleger a categoria intitulada "Identificação com o atendimento", em relação aos motivos que levaram os fisioterapeutas participantes da pesquisa à escolha de atuar junto a pessoas com paralisia cerebral. O conteúdo das respostas dos participantes evidenciou que a escolha dessa área de atuação ocorreu, num primeiro instante, por preferência pela área neurológica, e não necessariamente tratou-se de uma escolha em atuar junto às pessoas com paralisia cerebral. Embora algumas escolhas também possam ter ocorrido por a essa ser a primeira oportunidade de trabalho como fisioterapeuta, houve posteriormente uma identificação com a área, possibilitando que esses profissionais continuassem atuando junto à clientela citada. Constatou-se também, que a escolha dessa área de atuação não foi influenciada pela presença de pessoas com paralisia cerebral nas famílias desses profissionais, uma vez que prevaleceram as respostas indicando ausência de familiares nessa condição.

No que se refere a lidar com pessoas na condição de paralisia cerebral, prevaleceu, entre as respostas emitidas pelos fisioterapeutas, a descrição como "normal", sobre a qual foi criada a categoria "Normalidade", evidenciando que para os profissionais participantes, apesar dos atendimentos representarem um trabalho desafiador, tal desafio é visto por eles como sendo normal dentro de uma atuação em área neurológica. A normalidade conferida ao atender pessoas com paralisia cerebral refere-se, dessa forma, ao fato de que, para esses profissionais, esse tipo de clientela é "paciente neurológico", não sendo vista, necessariamente, como pacientes com paralisia cerebral, pois a fisioterapia é uma profissão que lida com distúrbios motores, conforme definição do COFFITO (2006): "[...] ciência da saúde que se ocupa do movimento humano, em estudá-lo, tratá-lo e a prevenir os distúrbios a ele relacionados."

Dessa forma, pode-se dizer que os profissionais pesquisados estavam preparados para o atendimento às pessoas com paralisia cerebral (paciente neurológico) no que se refere às 
dificuldades em obtenção de efeitos terapêuticos em curto prazo, pois os mesmos descreveram possuí ciência de que na área neurológica os resultados ocorrem em longo prazo.

Esse fato contrasta com a hipótese do pesquisador quanto aos possíveis sentimentos de frustração desses profissionais em relação a atenderem pessoas com paralisia cerebral, hipótese esta baseada nas afirmações de Basil (1995), quando cita que a falha em perceber a eficácia dos esforços empreendidos em conseguir realizar uma interação pode afetar tanto a criança com paralisia cerebral quanto as pessoas que a cercam, causando uma desmotivação em ambas para continuarem tentando a tarefa. Portanto, contrariando tais afirmações, os participantes pesquisados consideram o lidar com pessoas na condição de paralisia cerebral como normal para o esperado em um quadro de natureza neurológica, conforme certamente já estavam preparados para lidar pelo próprio curso de formação em fisioterapia, já que o mesmo privilegia o modelo médico e as questões biológicas, conforme afirma Ricieri (2003, citado por Braz, 2006) ao citar que: “[...] tanto o ensino quanto a prática da Fisioterapia ainda seguem os modelos adotados pela Medicina."

As "Limitações em termos de expectativas" definiram outra categoria na análise dos dados. De maneira geral, para os fisioterapeutas consultados, atender pessoas com paralisia cerebral significa esperar resultados em longo prazo e não ter expectativas de grandes alterações no quadro, dessa forma se configurando num desafio. Conforme mencionado anteriormente, o conhecimento a respeito das características que envolvem os quadros neurológicos já prepara os fisioterapeutas a respeito das limitações do referido tratamento. No entanto, esse conhecimento não impede que os atendimentos sejam vistos como desafiadores na medida em que as expectativas de melhoras são limitadas. Em decorrência disso, as mudanças obtidas pelos atendimentos tornam-se significativas para os profissionais pesquisados.

Quanto às impressões dos fisioterapeutas em relação ao que seus pacientes sentem quando mantêm contato físico (terapêutico) com os mesmos, de maneira geral prevaleceram respostas afirmativas, indicando que esses profissionais, embora lidem com pessoas na condição de paralisia cerebral grave, com ausência da linguagem verbal, conseguem perceber o que essas pessoas estão sentindo durante a realização da terapia. Esses dados suscitaram a criação da categoria "Empatia", cujo conceito é definido por Reeve (2006) como a capacidade de recriar a perspectiva de outra pessoa, experimentar o ponto de vista de alguém. Portanto, a hipótese do pesquisador sobre a existência de dificuldades vivenciadas pelos fisioterapeutas no atendimento às pessoas com paralisia cerebral - relacionada à ausência de linguagem verbal destes pacientes e a necessidade da criação de outras formas de comunicação entre ambos - não se confirmou, ainda que esta seja uma situação para a qual o fisioterapeuta comumente não é treinado durante sua formação, em razão da ênfase de seu curso ser no conhecimento biológico, em detrimento das questões da interação humana.

A predominância de respostas afirmativas quanto às habilidades em detectarem os sentimentos de seus pacientes com paralisia cerebral sugere uma adaptação dos profissionais às características de seus clientes. Essa adaptação remete à definição citada por Feijoo (2000), segundo a qual "o cuidado não é uma determinada forma de se relacionar, mas a condição de abertura às possibilidades de relações nos seus diferentes modos". Assim sendo, pode-se dizer que, na medida vem que esses profissionais colocam-se como disponíveis para ouvir as necessidades de seus clientes, ainda que sejam expressas de forma nãoverbal, ocorre uma adaptação do fisioterapeuta ao tipo peculiar de relação entre ele e seu paciente com paralisia cerebral, eliminando fatores que poderiam ser sentidos pelo profissional como dificuldades no atendimento.

As impressões dos fisioterapeutas sobre o que eles suscitam em seus pacientes durante os procedimentos fisioterapêuticos foram descritas e nomeadas por alguns profissionais. No entanto, não houve concordância entre os juízes e os pesquisadores quanto ao que foi respondido nesse item pelos participantes, por isso não será descritos nesta análise em razão de as reações apontadas não terem sido significativas para a mesma. Embora o objetivo da questão fosse identificar quais eram as impressões dos profissionais, as respostas fornecidas pelos participantes foram apenas se havia ou não a ocorrência das mesmas.

Quanto aos sentimentos experimentados pelos referidos fisioterapeutas no atendimento às pessoas com paralisia cerebral, as respostas, de uma forma geral, convergiram para sentimentos positivos, que culminaram na criação da categoria "Gratificante". Verificou-se pelos dados coletados uma predominância de sentimentos de gratificação em relação a atenderem essa clientela.

Apesar dos fisioterapeutas já possuírem conhecimento sobre as peculiaridades dos quadros neurológicos e das dificuldades encontradas no tratamento, os atendimentos foram descritos como desafiadores, tendo em vista que existem limites a 
serem transpostos, uma vez que os resultados podem ser reduzidos, obtidos de forma lenta e em longo prazo.

Portanto, o sentimento de que atender pessoas com paralisia cerebral é gratificante relaciona-se ao fato de quadros neurológicos serem vistos como desafios, e por isso os resultados terapêuticos, embora sejam reduzidos, alcançados de forma lenta e em longo prazo, fazem com que o atendimento seja vivenciado como gratificante pelos fisioterapeutas pesquisados, já que os resultados obtidos representam para eles a transposição de um desafio e superação.

Novamente observa-se um contraste entre este resultado e aqueles apontados pela literatura científica já descritos, quanto às dificuldades vividas pelos profissionais de saúde em seu cotidiano de trabalho nas relações com os clientes. Podemos supor que este contraste deve-se ao fato de que os participantes desta pesquisa são fisioterapeutas que atuam em média há sete anos e meio na profissão, e por isso talvez, de maneira geral, já tenham adquirido experiência e habilidades suficientes para atenderem pessoas com paralisia cerebral com maior tranquilidade em relação aos aspectos citados.

\section{Conclusão}

A análise de dados revelou que os fisioterapeutas consultados identificaram-se com o atendimento em área neurológica, mesmo quando esta não foi uma opção de trabalho escolhida previamente, fato que pode ter ocasionado nos profissionais uma melhor adaptação ao atenderem pessoas com paralisia cerebral, considerando também que durante os cursos de formação em fisioterapia os mesmos adquirem conhecimentos das peculiaridades dos quadros neurológicos, no que se refere à lenta evolução terapêutica dos casos. Esses elementos contribuem para que o atendimento a pessoas com paralisia cerebral seja visto como "normal" por estes profissionais, enquanto um tipo de trabalho cujas expectativas dos efeitos terapêuticos são para longo prazo.

$\mathrm{Na}$ medida em que as dificuldades em obter resultados são vistas como um desafio normal dentro do esperado em quadros neurológicos, os quais esses profissionais já se encontram tecnicamente treinados a enfrentar, o atendimento a pessoas com paralisia cerebral, então, é vivenciado como "gratificante", mesmo quando são alcançados resultados mínimos, pois qualquer mudança no quadro representa, para os profissionais de fisioterapia, uma superação de desafios por parte da pessoa com paralisia cerebral.
Embora o presente estudo tenha revelado tais resultados, o mesmo não pretende ser conclusivo, mas apenas lançar uma primeira reflexão que abra espaços para pesquisas mais aprofundadas em torno do tema. Um dos questionamentos emergentes do presente estudo refere-se às consequências da identificação da área neurológica com limitações em termos de expectativas, ou seja, sobre o quanto esta identificação pode ou não interferir em outros aspectos relacionados ao atendimento das pessoas com paralisia cerebral, que não necessariamente os aspectos relacionados aos sentimentos dos profissionais de saúde envolvidos, mas à própria qualidade dos atendimentos e/ ou dos esforços empreendidos na tarefa de atender.

Diante das considerações deste estudo, a psicologia - por ser uma profissão que trabalha com relações humanas - pode fornecer importantes contribuições no campo da saúde, atuando junto às equipes multi e interdisciplinares, especialmente no que se refere ao contexto da assistência humanizada. Cabe à Psicologia problematizar e propor amplo debate do como esse profissional, fisioterapeuta, consegue contemporizar e conciliar o desejo de reabilitar com a reabilitação lenta; como se estabelecem as relações entre profissional-curapaciente-família. Talvez Guareschi (1997) possa nos indicar a resposta, ao esclarecer que relação "é o ordenamento intrínseco de uma coisa em direção a outra [...] a idéia de incompletude, de precariedade, de historicidade".

Se a humanização da saúde é um apelo para o diálogo e a consideração dos aspectos subjetivos tanto de quem procura os serviços quanto de quem os presta, configurando-se, portanto, um cuidado com a pessoa humana, o psicólogo é o profissional cuja preparação técnica fornece os recursos para trabalhar com a promoção do desenvolvimento deste diálogo entre usuários, profissionais e instituições de saúde, sendo ele também o profissional de saúde que também carrega em sua prática o ouvir, o olhar, a intuição e a sensibilidade para detectar as demandas (de um ponto de vista mais privilegiado) - que podem ser traduzidas em "necessidades", em se tratando do ponto de vista daquele que necessita ser visto, compreendido e respeitado como sujeito na relação de diálogo enfatizada nos pressupostos da humanização dos serviços de saúde.

\section{Referências}

Basil, C. (1995) Os alunos com paralisia cerebral: desenvolvimento e educação. Em: C. Coll, P. Jésus \& P. Marchesi, (Orgs.). Desenvolvimento 
psicológico e educação: necessidades educativas especiais e aprendizagem escolar (pp. 253-271). Porto Alegre: Artes Médicas.

Braz, M. M. (2006). Educação integral: um modelo de ensino da fisioterapia baseado na física quântica. (Tese de Doutorado). Florianópolis: Universidade Federal de Santa Catarina.

Campos, D. C. (2003). Recém-formados em psicologia na atualidade e seus vinculos afetivos. (Tese de Doutorado). Campinas: Pontíficia Universidade Católica de Campinas - Instituto de Psicologia e Fonoaudiologia.

Conselho Federal de Fisioterapia e Terapia Ocupacional. (n.d.). Obtido em 19 de março de 2007 do World Wide Web: http://www.coffito.org.br.

Conselho Regional de Fisioterapia e Terapia Ocupacional de São Paulo. (n.d.). Obtido em 29 de agosto de 2006 do World Wide Web: http://www.crefito3.com.br.

Dalgalarrondo, P. (2000). Psicopatologia e semiologia dos transtornos mentais. Porto Alegre: Artmed.

Deslandes, S. F. (2004). Análise do discurso oficial sobre a humanização da assistência hospitalar. Ciência e Saúde Coletiva, 9(1), p. 7-14. Obtido em 26 de abril de 2007, do World Wide Web: http://www.scielo.br.

Deslandes, S. F., \& Ayres, J. R. C. M. (2005). Humanização em cuidado em saúde. [Editorial]. Ciência e Saúde Coletiva, 10(3), p. 510. Obtido em 26 de abril de 2007, do World Wide Web: http://www.scielo.br.

Diniz, D. (1996). Dilemas éticos da vida humana: a trajetória hospitalar de crianças portadoras de paralisia cerebral grave. Cadernos de Saúde Pública,
12(3), p. 345-355. Obtido em 26 de abril de 2007, do World Wide Web: http://www.scielo.br.

Feijoo, A. M. L. C. (2000). A escuta e a fala em psicoterapia: uma proposta fenomenológica-existencial. São Paulo: Vetor.

Guareschi, P. (1997). Qual a "prática" da psicologia da Abrapso? Em: Andréa V. Zanela, et cols. Psicologia e práticas sociais (pp. 11-16). ABRAPSO SUL: Porto Alegre

Martinez, J. E. \& Francisco, F. C. (2003). Um estudo de crianças da APAE de Adamantina (SP) portadoras de paralisia cerebral. Trabalho de Conclusão de Curso. Adamantina: Faculdades Adamantinenses Integradas.

Minayo, M. C. S. (1996). O desafio do conhecimento: pesquisa qualitativa em saúde. São Paulo: Hucitec/Abrasco.

Nogueira-Martins, M. C. F. (2004). Humanização das relações assistenciais: a formação do profissional de saúde. São Paulo: Casa do Psicólogo.

Reeve, J. M. (2006). Motivação e emoção. Rio de Janeiro: LTC.

Rocco, R. P. (1992). Relação estudante de medicinapaciente. Em: J. Melo Filho, (Org.). Psicossomática hoje. (pp. 42-52). Porto Alegre: Artes Médicas.

Takka, V. (1988). O relacionamento médico-paciente. Porto Alegre: Artes Médicas.

Vergara, S. C. (2005). Métodos de pesquisa em administração. São Paulo: Atlas.

Recebido em dezembro de 2007 Reformulado em novembro de 2008 Aprovado em janeiro de 2009

Sobre os autores:

Dinael Corrêa de Campos é doutor em Psicologia como Ciência e Profissão (2003) pela PUCCamp. Atualmente é professor assistente e supervisor de estágios do Curso de Psicologia da Universidade São Francisco, campus Pari/SP. Os principais interesses de pesquisa incluem Psicologia do Trabalho, Gestão de Pessoas e Recursos Humanos.

Márcia Guimarães Santos é psicóloga pela Universidade São Francisco, campus Pari/SP. Atualmente é membro da equipe técnica de uma instituição que abriga crianças em situação de risco social e pessoal, onde atua como psicóloga. 